\title{
Demand management in urine cytology: a single cytospin slide is sufficient
}

\author{
J L Burton, J R Goepel, J A Lee
}

\begin{abstract}
Aims-Current practice in most laboratories stipulates the preparation of duplicate slides for the analysis of urine cytology specimens. This study evaluates whether the duplicate slide is necessary.

Methods-Cytospin diagnosis was assessed in three ways. First, all urine cytology preparations from a single month in 1998 were reviewed; the two slides for each case were reported separately and then the two reports on each case were examined for disparity. Second, the slides from all urine cytospin cases indexed as "suspicious" or "malignant" in 1998 were reviewed similarly. Third, 48 cytospin slides from 24 cases were divided into two randomised groups, which were reported and the two reports compared. Finally, the frequency of repeat specimen collection in cases that were deemed inadequate for diagnosis was also assessed.
\end{abstract}

Results-The cases from a single month $(n=129)$ were representative of the annual workload and showed no discrepancies of the type: suspicious or malignant/ other. Of the 60 suspicious or malignant cases from 1998, there was no disparity in 50. The 10 cases with disparity were all suspicious on one slide and degenerate on the other. In the 24 randomised cases, there was no disparity in 21 . The remainder were reported as suspicious or malignant/inadequate (that is, degenerate or acellular). After a report of inadequate for diagnosis, repeat samples were received in only $15 \%$ of cases.

Conclusions-Using a single cytospin preparation causes minimal loss of clinically relevant information, but saves substantial resources $(\sim 40 \% /$ case $)$ A diagnosis of inadequate should prompt the collection of a repeat sample if the service is being used sensibly.

(f Clin Pathol 2000;53:718-719)

Keywords: urine cytology; cytospin; demand management

Department of Pathology, The Medical School, Beech

Hill Road, Sheffield

S10 2RX, UK

J L Burton

J R Goepel

J A Lee

Correspondence to: Dr Burton

email: j.1.burton@shef.ac.uk

Accepted for publication 7 January 2000

Exfoliative cytospin cytology of urine is an established sensitive and specific investigation in the diagnosis of carcinoma in situ and of primary and recurrent high grade transitional cell carcinoma of the bladder. ${ }^{1-5}$ It is also used rather indiscriminately to screen for lower grade lesions, although sensitivity is low in this setting. ${ }^{6}$ As a result, urine cytology gives rise to a large workload $(\sim 4 \%$ of the annual specimen total in our department), which is increasing with time.

In line with other laboratories, it has been our practice to examine two cytospin prepara- tions from each sample. However, published guidelines are lacking and most studies of urine cytospin cytology do not specify the number of preparations examined. Examination of a single cytospin would considerably reduce the work intensity associated with these specimens. Therefore, we investigated whether the duplicate cytospin preparation is necessary.

\section{Methods}

In our department, 1018 urine cytology specimens were reported in 1998, of which 60 (5.9\%) were either suspicious or malignant. We assessed the cytospin diagnosis in three ways. First, a single investigator reviewed all urine cytology specimens from a single month in 1998. There were 129 cases in the month chosen, representing $12.7 \%$ of the annual urine cytology workload. This month was representative of the annual sample $\left(\chi^{2}=0.03 ; \mathrm{p}=0.99\right)$ and contained eight cases $(6.2 \%)$, which were originally reported as suspicious or diagnostic of malignancy. The two slides from each case were individually categorised as malignant, suspicious, inflamed, negative, or inadequate for assessment (degenerate or acellular). The reports for the two separate slides from each case were then compared for disparity.

Second, the paired slides from all specimens indexed as suspicious or diagnostic of malignancy in 1998 were reviewed by three observers. Slides were allocated to one of the five diagnostic categories given above and reports were then compared for disparity.

Third, 48 slides from 24 cases were selected and divided into two randomised groups. There were eight malignant, eight negative, and eight degenerate cases. These were reported by the two observers who had not been responsible for case selection and again the two reports were compared for disparity.

Finally, we assessed the clinical response to a report of "inadequate". The laboratory database was searched to determine the proportion of such cases reported between January and June 1998 in which a repeat specimen (either urine cytology or a bladder biopsy) had been received.

\section{Results}

There was no difference between the reports for each individual slide in 124 of the cases (96\%). Of the five cases with disparity, three were reported as normal on one slide and inflamed on the other, and two were reported as acellular versus degenerate. There were no discrepancies of suspicious or malignant versus other.

Of the 60 positive cases reviewed by three observers, 50 showed no disparity between the two cytospin preparations. The 10 cases with disparity were all suspicious on one slide and 
Table 1 Histological diagnosis (where present) in cases with disparity between slides

\begin{tabular}{lll}
\hline Slide 1 & Slide 2 & Histological diagnosis \\
\hline Suspicious & Degenerate & G3 transitional cell carcinoma \\
Degenerate & Suspicious & None \\
Degenerate & Suspicious & Negative biopsył \\
Suspicious & Degenerate & Negative biopsy† \\
Suspicious & Degenerate & Chronic cystitis $\dagger$ \\
Suspicious & Degenerate & None \\
Degenerate & Suspicious & None \\
Degenerate & Suspicious & G3 transitional cell carcinoma $\neq$ \\
Suspicious & Degenerate & None \\
Suspicious & Degenerate & None
\end{tabular}

Interval between cytology and histology: ${ }^{\star}$ one month, ttwo months, $\ddagger 12$ months.

degenerate on the other. Of these cases, two patients have subsequently been diagnosed as having high grade transitional cell carcinoma (table 1).

Of the 24 randomised cases reviewed by two observers, one observer showed no disparity between the slides. The other observer showed no disparity in 21 cases. Of the remaining three cases, two were reported as suspicious/ inadequate and one as malignant/inadequate.

In 1998,346 cases were reported as inadequate ( $34 \%$ of all cases). Of these, 173 were reported in the six month period evaluated. A repeat specimen was received in only 25 cases $(15 \%)$. In the 25 repeats, the cytological diagnoses were malignant (five), suspicious (one), inflamed (four), negative (five), and inadequate (six). The remaining four cases had a biopsy, which was normal in three and showed a leiomyoma in one. The mean time to repeat specimen collection was 4.7 months (range, $0-17$ ).

\section{Discussion}

Urine cytology is a controversial investigation that has gained few enthusiastic supporters among pathologists. High grade lesions can be detected fairly reliably, ${ }^{1-5}$ although there is a false negative rate of approximately $20 \%$. $^{7}$ However, the relatively normal cytological appearance of low grade tumours, coupled with a generally low cell yield, results in an overall sensitivity of only about $30 \%$ in this setting. ${ }^{67}$ Thus, urine cytology is not an appropriate investigation for general screening purposes, but can be a helpful adjunct investigation in high risk groups or in follow up.

However, urine cytology is a popular investigation among clinicians, ${ }^{8-11}$ who often use it indiscriminately and with little apparent awareness of its limitations. Indeed, in our experience, the expansion of urine cytology typifies the unfunded and sometimes irrational development of services within the National Health Service. Since a clinicopathological meeting five years ago (in which none of the authors were involved), we have seen an explosive expansion in the number of samples: a 10-fold increase initially, which has continued to rise more slowly over the past four years. This is mainly because of an arrangement whereby patients seen in the urology outpatient department with recent onset haematuria undergo a "one stop" panel of investigations that includes urine cytology. Despite requests for a proper business case, we have been unable to reverse this trend.
In view of the substantial and increasing workload that these samples represent, we undertook our study as an exercise in partial demand management. The results demonstrate that in most cases, no extra information is provided by the duplicate cytospin slide. In those positive cases with disparity, the nondiagnostic slide was inadequate. In a service that was sensibly using the information provided by cytospin cytology, a diagnosis of inadequate should generate a prompt repeat specimen in a substantial number of cases. The fact that this happened in only $15 \%$, with a mean time delay of 4.7 months, indicates the failings of the system currently in place. Nevertheless, as a result of our study, we have rationally reduced the laboratory and diagnostic workload for this specimen type, in a way that also applies to systems where the test is being used appropriately. The calculated laboratory workload reduction for each case is approximately $40 \%$ (from 21 to 13 Welcan units) and in our hands the medical reporting time is similarly reduced (most of our time is spent looking at slides because we use proforma reporting with these specimens).

A potential worry associated with using a single cytospin is that it might increase the number of repeat specimens sent. However, our findings indicate that this would be necessary in only a minority of appropriately referred cases and, in any event, this would be preferable to the current misuse of the service. Our next step will therefore be to use the information gathered in our study to extend our partial demand management to full demand management, by instigating a local reappraisal of the use of urine cytology in the investigation of patients presenting with recent onset haematuria.

In conclusion, our study shows that urine cytology can be assessed adequately with a single cytospin preparation (resulting in a substantial reduction in workload) and that in the setting of an appropriate request for urine cytology an inadequate specimen should prompt a repeat collection.

1 Bondhus M, Leif R, Bobbitt D, et al. Centrifugal cytology: a new technique for urine cytology. Urology 1986;28:432-3.

2 Dhundee J, Rigby HS. Comparison of two preparatory techniques for urine cytology. F Clin Pathol 1990;43:10345.

3 Loh CS, Spedding AV, Ashworth MT, et al. The value of exfoliative urine cytology in combination with flexible cystoscopy in the diagnosis of recurrent transitional cell carci-

4 Stonehill WH, Goldman HB, Dmochowski RR. The use of urine cytology for diagnosing bladder cancer in spinal cord urine cytology for diagnosing bladder cancer
injured patients. F Urol 1997;157:2112-14.

5 Murphy WM. Current status of urinary cytology in the Murphy WM. Current status of urinary cytology in the
evaluation of bladder neoplasms. Hum Pathol 1990;21:88696.

6 Goldstein MM, Messing EM. Screening of bladder cancer. In: Syrigos KN, Skinner DG, eds. Bladder cancer: biology, diagnosis and management. Oxford: Oxford University Press, 1999:57-70.

7 Stein JP, Skinner DG. Clinical presentation and investigation of bladder cancer. In: Syrigos KN, Skinner DG, eds. Bladder cancer: biology, diagnosis and management. Oxford: Oxford University Press, 1999:167-80.

8 Kern WH, Bales CE. Quantitative studies of urine cytology. Am f Clin Pathol 1969;51:225-8.

9 Matzkin H, Moinuddin SM, Soloway MS. Value of urine cytology versus bladder washing in bladder cancer. Urology 1992;39:201-3.

10 Tut VM, Hildreth AJ, Kumar M, et al. Does voided urine cytology have biological significance? Br f Urol 1998;82: 655-9.

11 Murphy WM, Crabtree WN, Jukkola AF, et al. The diagnostic value of urine versus bladder washing in patients with bladder cancer. F Urol 1981;126:320-2. 